UCRL-ID-119154

\title{
Finite Element Radiation Transport in One Dimension
}

\author{
J. F. Painter
}

May 9, 1997

This is an informal report intended primarily for internal or limited external diatribution. The opinions and concluoions atated are those of the author and may or may not be those of the Laboratory.

Work performed under the aupices of the U.S. Department of Energy by the Lawrence Livermore National Laboratory under Contract W-7405-Eng-48. 


\section{DISCLAMMER}

This document wis propared as an account of wots sponsored by an agency of the United States Government. Neither the Unibed States Government nor the Univerdty of Callfornia nor any of their einployees, makes any warranty, expreses or implied, or meumes any lezal liablity or respondbility for the sccurncy, completences, or veefulness of any information, epperratus, product, or process disclosed, or represents that its ue would not infinge privelely owned rights. Reference hereln to any specific commercial product, process, or service by trade name, trademark, menufacture, or otherwise, does not necesearily constitute or imply its endorsement, recommendation, or favoring by the United States Covernment or the Universtly of California. The views and opinions of authors expresced herein do not necesearily state or refiect thoee of the Unibed States Government or the Univerdity of California, and shell not be used for advertiding or product endorsement purposes.

This report hes been reproduced directly from the best available copy.

Available to DOE and DOE contractors from the Office of Sctentific and Technical Informution P.O. Box 62, Oak Ridge, IN 37831

Prices available from (615) 576-8401, FTS 626-8401

Avallable to the public from the

National Techrical Information Service

U.S. Depertment of Commerce 5285 Port Royal Rd., Springfield, VA 22161 


\title{
Finite element radiation transport in one dimension
}

\author{
Jeffrey F. Painter \\ Lawrence Livermore National Laboratory
}

A new physics package solves radiation transport equations in one space dimension, multiple energy groups and directions. A discontinuous finite element method discretizes radiation intensity with respect to space and angle, and a continuous finite element method discretizes electron temperature in space. A splitting method solves the resulting linear equations. This is a one-dimensional analog of Kershaw and Harte's two-dimensional package. This package has been installed in a two-dimensional inertial confinement fusion code, and has given excellent results for both thermal waves and highly directional radiation. In contrast, the traditional discrete ordinate and spherical harmonic methods show less accurate results in both cases. (U)

\section{Introduction}

Most radiation transport methods have accuracy problems in some regimes. Sn (discrete ordinate) methods do a decent job of tracking highly directional radiation, but need ad hoc corrections to provide acceptable results on thermal waves. Spherical harmonic methods are inaccurate for streaming radiation.

Some time ago Kershaw and Harte (1993) wrote a two-dimensional radiation transport package using a discontinuous finite element method and proposed a one-dimensional package. This two-dimensional code proved successful for a wide range of problems, so I wrote another code using the proposed one-dimensional method.

The next section will describe the numerical methods used. Then this paper will discuss interfacing this one-dimensional finite element code to twodimensional finite difference codes. The third section will present calculations by this code and older radiation transport codes. For both streaming radiation and thermal waves, this discontinuous finite element method gives the best results. For many people the dependable accuracy of this method will prove worth the greater running time.

\section{Numerical methods}

The basic approach is a Galerkin finite element method, bilinear in space and direction. Geometry can be spherical or planar. A radiation transport equation has upwinded discontinuous elements, and an electron temperature equation has continuous elements. The resulting linear equations are solved by a modified splitting of the intensity and temperature parts. There is an optional Newton iteration for problems where the nonlinearity of the Planckian function is significant over a single time step - a feature not in the twodimensional code.

Following Kershaw and Harte (1993), we begin with an equation for the radiation intensity $I$ :

$$
\frac{1}{c} \frac{\partial I}{\partial t}+\frac{d l}{d k}=\rho \kappa\left(I_{p}-I\right)
$$

where $k$ is distance along the direction of the radiation, $I_{p}=I_{p}\left(T_{e}\right)$ is the Planckian intensity for the electron temperature $T_{e}, \rho$ is density, $x$ is opacity, $t$ is time, and $c$ is the speed of light.

For simplicity we can discretize time with the backwards Euler method, hiding time dependence in a source:

$$
c \frac{d I}{d k}=\eta(S-I)
$$

with

$$
S=\frac{c \rho \kappa \Delta t I_{p}+I(t-\Delta t)}{c \rho \kappa \Delta t+1}
$$

and

$$
\eta=c \rho \kappa+1 / \Delta t
$$

Describe the radiation direction by an angle cosine $\mu$, the cosine of the angle with a ray pointing directly outward or right.. If we separately discretize $\mu$ and the spatial distance $r$, we have rectangular zones in $(r, \mu)$ space. Each zone has four nodes and vice versa, as shown in the following diagram. In each such zone $(i, \alpha)$, approximate $I$ by a bilinear function

$$
I(r, \mu)=\sum_{J=1}^{4} I_{i, \alpha}^{J} \phi_{i}^{J}(r) \psi_{\alpha}^{J}(\mu)
$$

where $J$ is a node number and $\phi$ and $\psi$ are linear functions, taking values 0 and 1 near nodes. It is important to note that every $(r, \mu)$ node has four values of $I, I_{i, \alpha}^{J}$. There is one value for each zone $(i, \alpha)$ adjoining that node. Thus we explicitly allow for discontinuous solutions such as streaming radiation. 


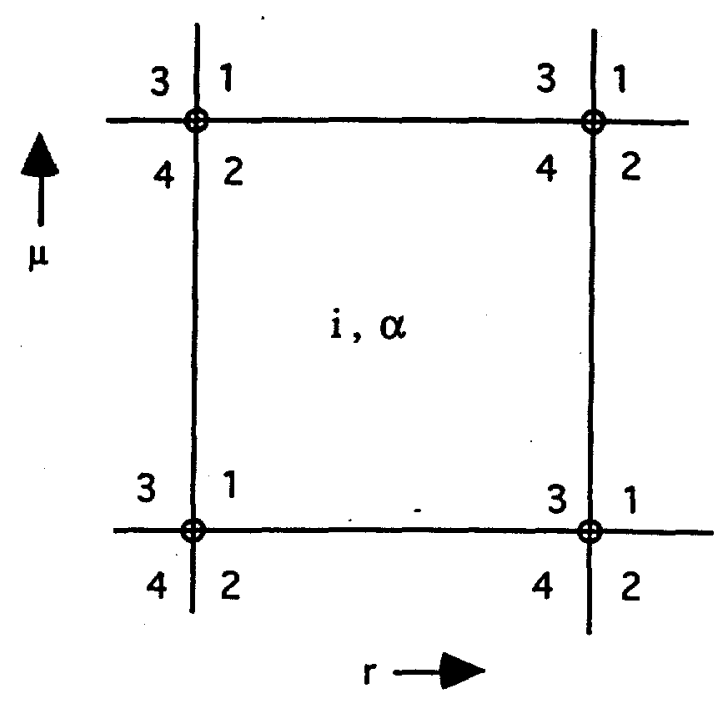

The other equation solved in this model determines the electron temperature:

$$
C_{\nu} \frac{\partial T_{e}}{\partial t}+\int_{0}^{\infty} \int_{-1}^{1} c \rho x\left(I_{p}-I\right) d \mu d v=0
$$

where $v$ is the frequency and $C_{v}$ is the specific heat. Like the intensity $I(r, \mu)$, we can approximate the temperature $T_{e}(r)$ with linear basis functions $\phi(r)$ taking values 0 and 1 at nodes. Unlike intensity, it is reasonable to require temperature to be continuous. Then in the $i$-th $r$ zone, $T_{e}$ is

$$
T_{e}(r)=T_{e}\left(r_{i}\right) \phi^{1}(r)+T_{e}\left(r_{i+1}\right) \phi^{2}(r)
$$

Following the usual method of constructing Galerkin equations, multiply an arbitrary basis function by each of the equations (2) and (6), substituting the approximations (5) and (7).

We can exploit an ambiguity implicit in this recipe: we have specified the values of the bilinear functions in the zones but not on the zone boundaries. These values matter in a discontinuous finite element method because the derivative in (2) is a delta function where $I$ is discontinuous; hence it picks out boundary values.

A natural way to choose boundary values for basis functions is upwinding. On a zone boundary, set the basis function to its limiting value if the radiation is entering the zone or tangential, 0 if the radiation is leaving the zone. This upwinding scheme ensures stability without requiring a lower order of accuracy.

In slab geometry, $\mu$ is constant in the direction of radiation, so the only upwind zone boundaries are $r$ boundaries. If $\mu>0$, the radiation is moving right and $r_{i}$ is upwind of $r_{i+1}$; if $\mu<0$ the radiation is moving left and $r_{i+1}$ is upwind of $r_{i}$. In spherical geometry, $\mu$ determines the upwind $r$ zone boundaries the same way. Along the direction of radiation, $\mu$ is always increasing, so $\mu_{\alpha}$ is always upwind of $\mu_{\alpha+1}$.

Using the fact that

$$
\frac{d}{d k}=\mu \frac{d}{d r}+\frac{1-\mu^{2}}{r} \frac{d}{d \mu}
$$

in spherical geometry, and

$$
\frac{d}{d k}=\mu \frac{d}{d r}
$$

in slab geometry, it is straightforward to compute the resulting Galerkin equation for intensity. After expanding and integrating across zone boundaries but before the other expansions and evaluations, it is

$$
\begin{aligned}
& 0=\int_{r_{i}}^{r_{i+1}} \int_{\mu_{\alpha}}^{\mu_{\alpha+1}} \phi_{i}^{J}(r) \psi_{\alpha}^{J}(\mu) \\
& +\left(c \frac{d I}{d k}-\eta(S-I)\right) d \mu r^{2} d r \\
& +\int_{r_{i}}^{r_{i+1}}\left(1-\mu_{\alpha}^{2}\right) \phi_{i}^{J}(r) \psi_{\alpha}^{J}\left(\mu_{\alpha}\right) c \\
& \sum_{J^{\prime} \in 1,3}\left(I_{i, \alpha}^{J^{\prime}}-I_{i, \alpha-1}^{J^{\prime}+1}\right) \phi_{i}^{J^{\prime}}(r) r d r \\
& +\int_{\mu_{\alpha}}^{\mu_{\alpha+1}} \mu d \mu \psi_{\alpha}^{J}(\mu) c \\
& \left\{\begin{array}{c}
r_{i+1}^{2} \phi_{i}^{J}\left(r_{i+1}\right) \sum_{J^{\prime}=3,4}\left(I_{i+1, \alpha}^{J^{\prime}-2}-I_{i, \alpha}^{J^{\prime}}\right) \psi_{\alpha}^{J^{\prime}}(\mu) \\
r_{i}^{2} \phi_{i}^{J}\left(r_{i}\right) \sum_{J^{\prime}=1,2}\left(I_{i, \alpha}^{J^{\prime}}-I_{i-1, \alpha}^{J^{\prime}+2}\right) \psi_{\alpha}^{J^{\prime}}(\mu) \\
\text { if } \alpha>\frac{1}{2} n_{\mu}
\end{array}\right.
\end{aligned}
$$

for spherical geometry, and

$$
\begin{aligned}
& 0=\int_{r_{i}}^{r_{i+1}} \int_{\mu_{\alpha}}^{\mu_{\alpha+1}} \phi_{i}^{J}(r) \psi_{\alpha}^{J}(\mu) \\
& \left(c \frac{d I}{d k}-\eta(S-I)\right) d \mu d r \\
& +\int_{\mu_{\alpha}}^{\mu_{\alpha+1}} \mu d \mu \psi_{\alpha}^{J}(\mu) c \\
& \left\{\begin{array}{c}
\phi_{i}^{J}\left(r_{i+1}\right) \sum_{J^{\prime}=3,4}\left(I_{i+1, \alpha}^{J^{\prime}-2}-I_{i, \alpha}^{J^{\prime}}\right) \psi_{\alpha}^{J^{\prime}}(\mu) \\
\text { if } \alpha \leq \frac{1}{2} n_{\mu} \\
\phi_{i}^{J}\left(r_{i}\right) \sum_{J^{\prime}=1,2}\left(I_{i, \alpha}^{J^{\prime}}-I_{i-1, \alpha}^{J^{\prime}+2}\right) \psi_{\alpha}^{J^{\prime}}(\mu) \\
\text { if } \alpha>\frac{1}{2} n_{\mu}
\end{array}\right.
\end{aligned}
$$

for slab geometry. In equations (10) and (11), $J$ is the node index as shown in the diagram, and $n_{\mu}$ is the number of discretized directions $\mu_{\alpha}$, assumed to be even and equally spaced.

The discretized temperature equation is simpler because we are modeling electron temperature as continuous. With the Planckian linearized about a temperature $T_{e}{ }^{I}$, time discretized by the backwards Euler method, and before substituting (5) and (7), the part of the equation in zone $i$ is: 


$$
\begin{aligned}
& \frac{C_{v}}{\Delta t} \int_{r_{i}}^{r_{i+1}}\left(T_{e}-T_{e}^{0}\right) \phi_{i}^{J}(r) r^{2} d r= \\
& \int_{r_{i}}^{r_{i+1}} \phi_{i}^{J}(r) r^{2} d r \int_{0}^{\infty} \int_{-1}^{l} c \rho x d \mu d v \\
& \quad\left(1-I_{p}\left(T_{e}^{I}\right)-\frac{d I_{p}\left(T_{e}^{I}\right)}{d T}\left(T_{e}-T_{e}^{I}\right)\right)
\end{aligned}
$$

for spherical geometry, and the same without the $r^{2}$ factors for slab geometry. Here $T_{e}^{0}=T e(t-\Delta t)$., and we are assuming that the specific heat varies little within a zone. Because of the continuity of $\mathrm{Te}$, the actual basis functions are $\phi_{i-1}^{2}+\phi_{i}^{1}$, so pairs of equations (12) must be added to get the full Galerkin equation with the correct basis functions.

The Planckian function also appears in $(10,11)$ through $S=S\left(T_{e}\right)$, defined by (3). Linearize that as in (12). Then $(10,11)$ and (12) are all linear equations.

To make them easy to solve, first note from (12) that the equation for the basis function $\phi_{i}^{1}+\phi_{i-1}^{2}$ involves the unknowns

$$
\begin{aligned}
& T_{e i}^{1}=T_{e i-1}^{2}, T_{e i}^{2}, T_{e i-1}^{1} \\
& I_{i, \alpha}^{1,2}, I_{i-1, \alpha}^{3,4}, I_{i, \alpha}^{3,4}, I_{i-1, \alpha}^{1,2}
\end{aligned}
$$

Considering the intensities as given, this is a tridiagonal matrix. To simplify the solution, we "lump" the matrix. That is, drop the off-diagonal elements (the coefficients of the second two unknowns in each row of (13)), and instead add them into the diagonal.

Inspection of $(10,11)$ shows that the equation for the basis function $\phi_{i}^{1} \psi_{\alpha}^{1}$ involves the unknowns

$$
\begin{aligned}
& I_{i, \alpha}^{J}, I_{i, \alpha-1}^{2}, I_{i, \alpha-1}^{4}, I_{i \pm 1, \alpha}^{\{1,3\}}, I_{i \pm 1, \alpha}^{\{2,4\}}, \\
& T_{e i}^{2}, T_{e i}^{1}
\end{aligned}
$$

with similar lists for the basis functions that are one at other nodes. The superscripts in braces correspond to the \pm in subscripts. Consider the temperatures as given, and order the intensities to correspond to the upwinding scheme. Then (14) shows that the matrix is blocktriangular. The blocks are $4 \times 4$, corresponding to the four nodes in a zone. There is a diagonal for the intensities in zone $(i, a)$, and off-diagonals below it for zones $(i, \alpha-1)$ and $(i \pm 1, \alpha)$.

$d I^{J} / d T_{e} J^{\prime}$, with $I$ and $T_{e}$ at the same spatial node, and all angles and angular nodes for $I$. Then for $I$ in (12) substitute $I+\left(d I / d T_{e}\right)\left(T_{e}-T_{e}^{I}\right)$. The temperature equation remains diagonal because of the nodal values used for $d l / d T_{e}$. WiThe temperature coupling term $S-I$ in $(10,11)$ couples intensities at all 4 nodes in a $(r, \mu)$ zone to temperatures at both nodes of the $r$ zone. We "lump" that part of the matrix just as we lumped the temperature matrix. Drop the coefficients that couple different spatial nodes, and add those coefficients to the diagonal.

We solve the whole linear system by a splitting method, alternately solving the lumped temperature and intensity matrices. As they are diagonal and triangular, they can be solved quickly, and matrix elements assembled on the fly.

This method is an option in the present code, but the splitting iteration can be sped up by making one more change. When the matrix $(10,11)$ is assembled and solved for $l$, given a temperature $T_{e}^{l}$, also solve for th a part of the intensity equation included in the temperature equation in this manner, convergence often takes only one or two iterations. That is the case, for example, with the test problems described later in this paper.

Another elaboration in the code is to wrap a Newton iteration around the linear iteration, thus providing for those problems where the Planckian function is nonlinear enough to matter.

\section{Interfacing}

It is always a substantial job to correctly insert a physics package in a large comprehensive physics code. Two of the more interesting issues here are what happens when a finite element package is interfaced to finite difference codes, and what happens when a package using spherical geometry must be interfaced to a larger code based on cylindrical geometry.

\section{Nodal to zonal and back}

In the first case, we must convert between finite element variables, which model physical quantities at all points, based on nodal values; and finite difference variables, which are defined only at particular points, normally zone centers. This is an especially critical problem for electron temperature, which serves as both an input and an output for our radiation transport package. For this the one-dimensional code follows the lead of Kershaw and Harte's two-dimensional code. Similar ideas are used for specific heats and other quantities.

To ensure energy conservation, this code converts only electron energies, not temperatures, between finite difference and finite element formulations. Energies are transformed into temperatures and back when needed. For input to this code, we must compute two nodal electron energies for each zone. That is, we must partition each zonal energy between the two basis functions $\phi^{J}(r)(J=1,2)$ with support on that zone. Usually this partition is done in proportion to the corresponding volumes

$$
V_{i}^{J}=\int_{r_{i}}^{r_{i+1}} \phi^{J}(r) r^{2} d r
$$

The previous cycle's electron energy partition is sometimes used instead when necessary to ensure a positive energy.

The conversion method described in the above paragraph is diffusive: a zonal temperature will affect two nodal temperatures, which then will affect three zonal temperatures. To control this effect, the natural approach is to convert only the last cycle's change in energy, rather than all of the electron energy. We do that on input to this package, but for stability we convert all of the energy on output. 
For the transformation from nodal temperatures to nodal energies, we begin with the old zonal energy $E^{z}$ and the corresponding zonal temperature $T_{e}{ }^{z}$. Upon computing the new nodal temperatures $T_{e}$ in $(7,12)$ we use the zonal specific heat $C_{v}$ and nodal volume fractions

$$
v f^{J}{ }_{i}=\frac{V_{i}^{J}}{V_{i}^{1}+V_{i}^{2}}
$$

to get the new nodal energies:

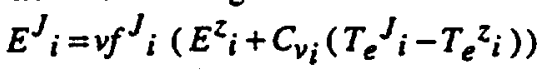

The energy to temperature conversion is done simply by solving this equation (summed over both sides of a node) for $T_{e}{ }^{2} i=T_{e}{ }_{i+1}$.

To convert nodal energies from the finite element model to zonal energies for finite difference use, one simply has to sum the nodal energies found by (17).

\section{Geometric adjustments}

The second interesting interface problem occurs where this one-dimensional code must be interfaced to a two-dimensional code. Suppose we want to solve a onedimensional spherical problem with a two-dimensional code based on cylindrical geometry. We can approximate a wedge of the sphere using appropriate radii and axial distances in the cylindrical geometry. Where the cylindrical radii are zero, the axial distance serves as the spherical wedge's radius. But the zonal volumes will be a little off because the curved zonal faces in spherical geometry are approximated with flat ones in cylindrical geometry.

So the main geometric problem in interfacing the two types of codes lies in deciding which volume to use when. Most zonal quantities need a geometric adjustment when converted between the onedimensional package and the host code. For example, to convert from the finite element representation of intensity to zonal radiation energies for finite difference use, we first use the spherical volume fractions $(15,16)$ to compute the average intensity within a (radius, angle) zone. Then we integrate over angle and multiply by the approximate zonal volume from cylindrical coordinates.

\section{Example calculations}

We ran this code on two limiting cases that have analytic solutions in some sense. For comparison, we ran the same problems using two other methods that keep track of the directionality of the radiation: $\mathrm{Sn}$ (discrete ordinates) and $\mathrm{Pn}$ (spherical harmonic).

\section{Thermal wave}

The first test problem is a thermal wave. In slab geometry, there is a uniform material with a high opacity; we used a perfect gas $(\gamma=5 / 3)$ with opacity fixed at $10^{4}$, and density set to 1 . Initially the material is cold, but a thermal wave is set off by fixing the temperature at one side to 1 .

Equations $(1,6)$ have an analytic solution for $\rho \kappa \rightarrow \infty$, in terms of

$$
\xi=\frac{z}{\sqrt{a t}}
$$

where $\mathrm{z}$ is the distance across the slab and

$$
a=\frac{4}{3} \frac{c \alpha}{\rho \kappa C_{v}}
$$

where $\alpha$ is a conversion constant. For $\xi>\xi_{0}-\sqrt{2 / 3}$, $T_{e}=0$, and for $\xi_{<} \xi_{0}$,

$$
T_{e}=\left(1-\frac{\xi}{\xi_{0}}\right)^{1 / 3}\left(1+O\left(\xi_{0}-\xi\right)^{4 / 3}\right)
$$

The following plots show the electron temperature as a function of radius at various times, as computed by the three numerical methods. This is done for 30 zones in all cases, and both with 30 angles (or moments for $\mathrm{Pn}$ ) and with 4 angles. This analytic solution is overlain.
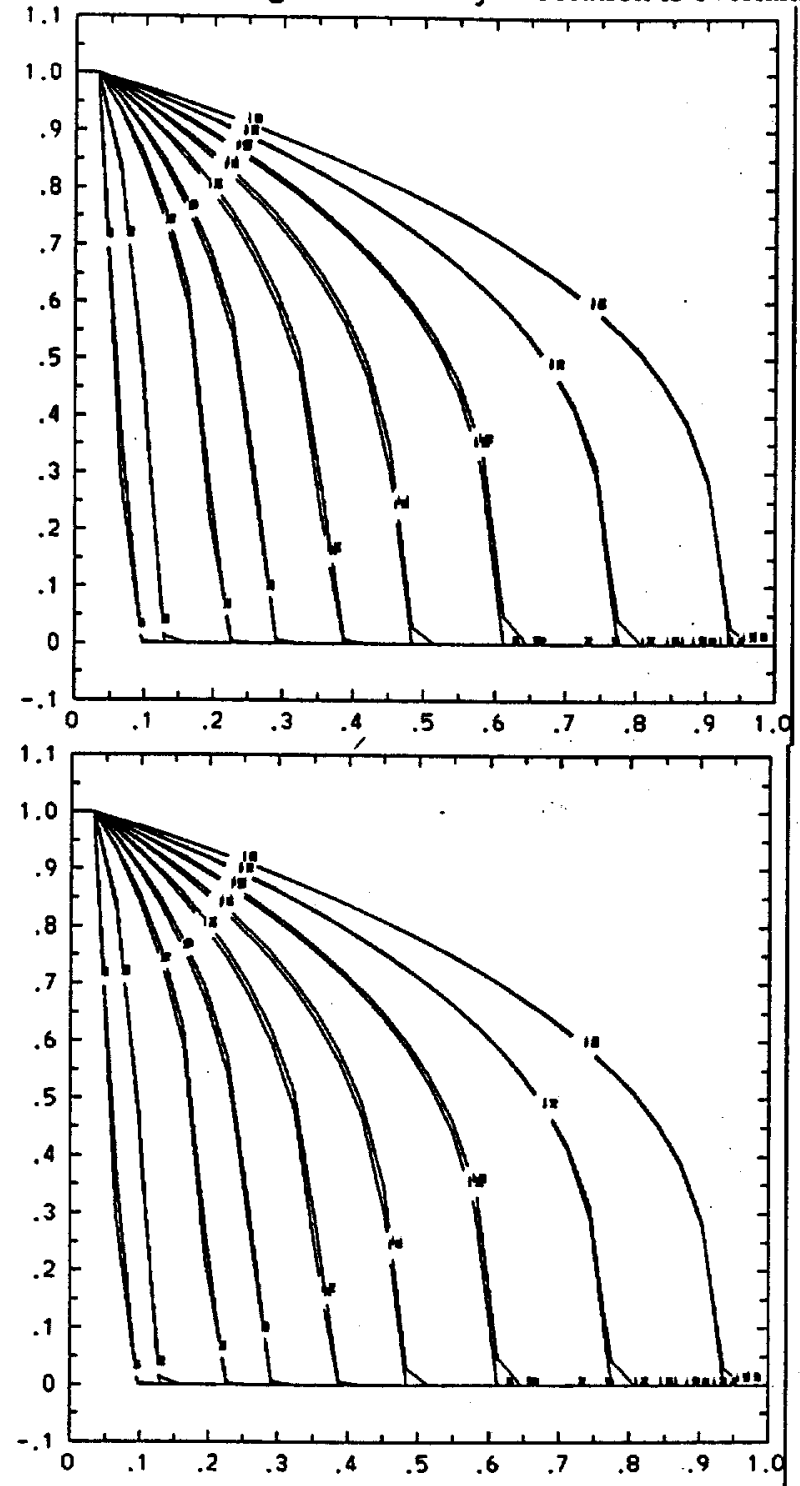

Figure 1. Thermal wave at various times, electron temperature vs. distance; analytic and finite element computational solutions. Upper plots: 4 computational angles; lower plots: 30 angles.

The finite element solution is very close to the analytic solution, regardless of the number of angles. 
-This is probably because, as shown in Kershaw and Harte (1993), the limit as $\rho \kappa \rightarrow \infty$ of the finite element solution of equations $(1,6)$ is the same as a finite element solution of the limit as $\rho K \rightarrow \infty$ of equations $(1,6)$. In this limit of the analytic equations, intensity depends on direction only as a simple quadratic in $\mu$. So it is not surprising that few angles are needed to get the electron temperature right.

Similarly, the Pn solutions are the same for either number of moments. The wave speed is off by a zone, or occasionally two zones. One factor that may have affected its accuracy here is that the $\mathrm{Pn}$ code was run with a different time step control algorithm than the others, and it had much larger time steps than the others for this problem.

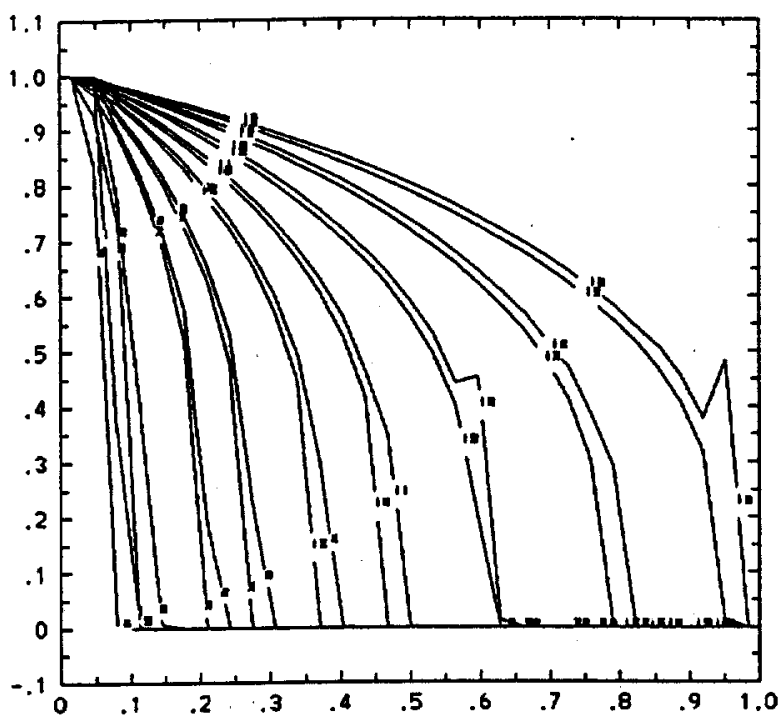

Figure 2. Thermal wave at various times,electron temperature vs. distance; analytic and $\mathrm{Pn}$ computational solutions, 30 moments.

The Sn method has a reputation for being inaccurate on thermal waves. That it performed as well as it did in these tests is probably because the Sn code included a Wilson-Lund-Castor correction factor designed for optically thick zones. See Lund and Wilson (1980). Nevertheless, the Sn method gets the wave speed little less accurately then the finite element method for a large number of angles. For a small number of angles. the $\mathrm{Sn}$ method is a full zone off. Probably the correction factor is less accurate when it has to represent a wider range of angles with a single number.

Table 1. Running time for thermal wave problem, problem time 0 to 150, on a Sun SPARC-10.

\begin{tabular}{lrrc}
\hline method & cycles & $\begin{array}{c}\text { CPU } \\
\text { minutes }\end{array}$ & $\begin{array}{c}\text { milliseconds } \\
\text { per zone- } \\
\text { angle-cycle }\end{array}$ \\
d.f.e. & 1219 & 14.1461 & 0.77 \\
Pn & 82 & 0.2189 & 0.18 \\
Sn & 1430 & 1.9611 & 0.09
\end{tabular}

The $\mathrm{Pn}$ method ran fast because of its different time step control algorithm. The greater running time per cycle for the finite element method is probably because it has four times as much intensity data per zone-angle. This method solves many four-by-four matrix blocks every cycle, and a four-by-four matrix solve takes about 17 times as much time as a one-by-one solve.
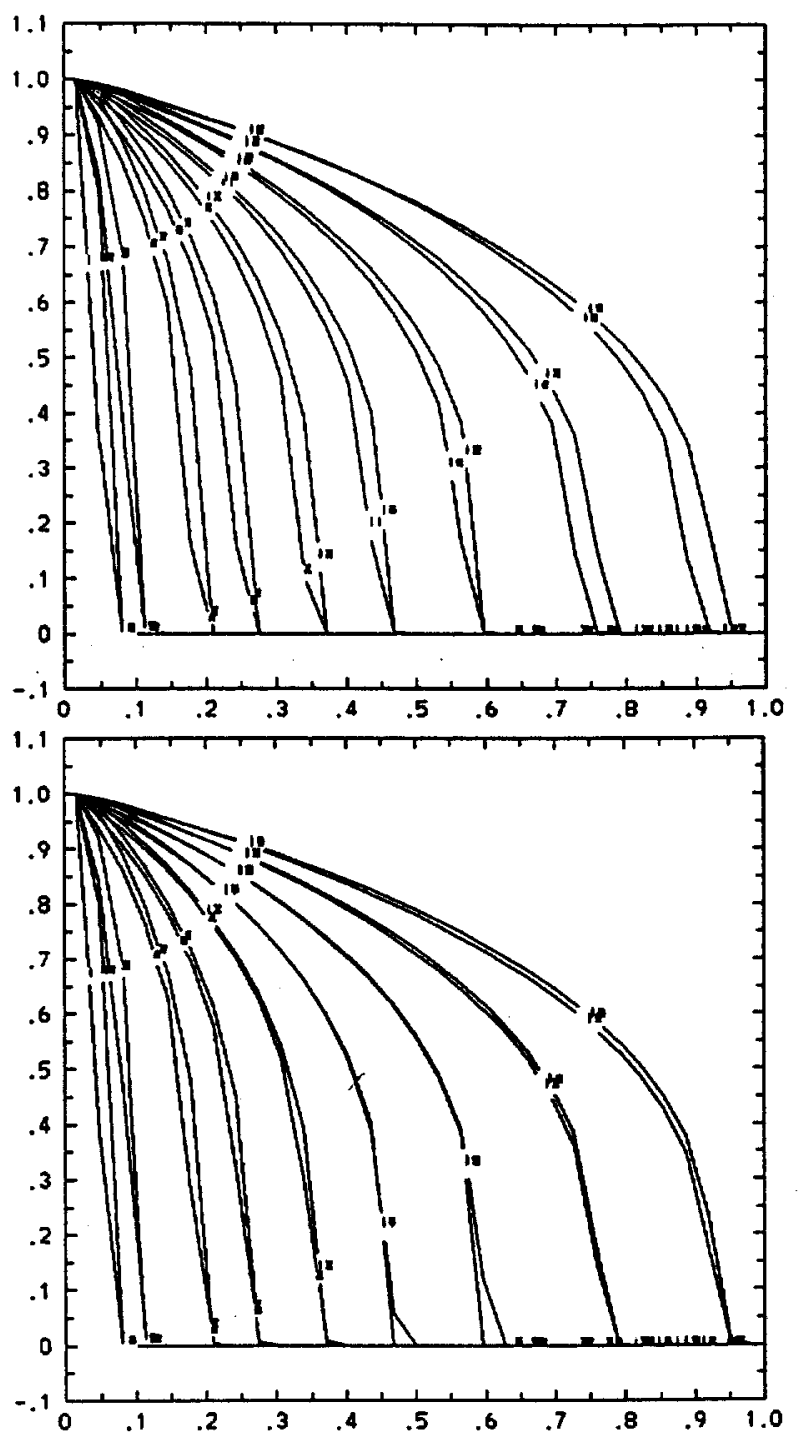

Figure 3. Thermal wave at various times, electron temperature vs. distance; analytic and $\mathrm{Sn}$ computational solutions. Upper plots: 4 computational angles; lower plots: 30 angles.

\section{Spherical Streaming Problem}

The second test problem tests computation of streaming radiation in spherical geometry. A sphere is maintained at a constant temperature and radiates into a vacuum in spherical geometry. We compute a steadystate solution. The sphere is suddenly turned off (to zero temperature). Each point in the vacuum will have a constant intensity until enough time has passed for light to travel from the nearest point of the sphere; then intensity will drop until the time at which light would come from the farthest visible point of the sphere. 
The analytic solution is a simple geometric calculation. At any radius and direction, the intensity is zero or a positive constant; it is positive if a ray in that direction hits the sphere at a distance greater than $c(t-$ $\left.t_{0}\right)$, where $t_{0}$ is the time at which the source is turned off.

In this problem it is interesting to look at both the radiation energy density as a function of radius, and the intensity as a function of angle, at a fixed radius. All runs had 40 equally spaced zones and 30 angles or moments.

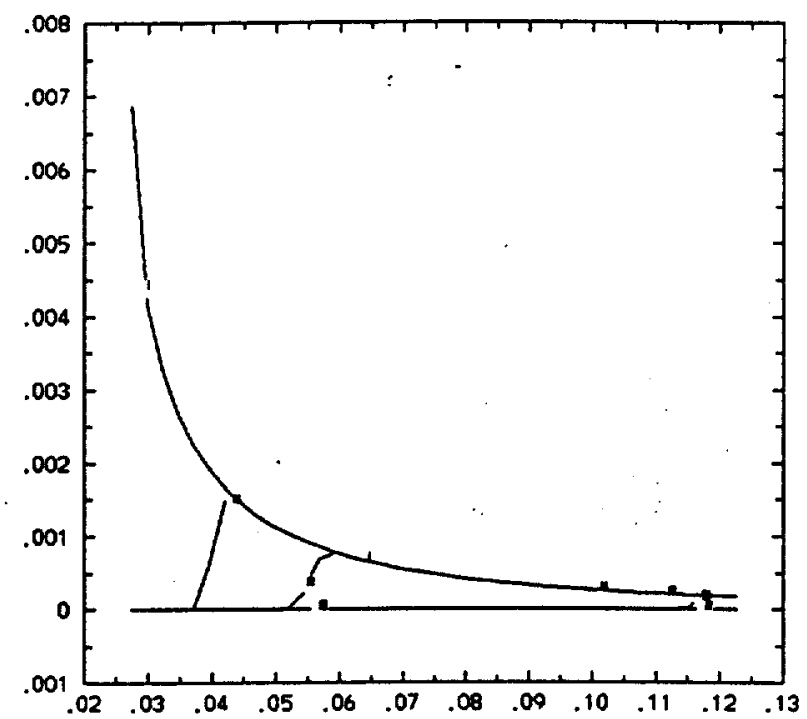

Figure 4. Spherical Streaming problem, radiation energy density vs. radius; analytic solutions at steady-state and various times after source turns off.

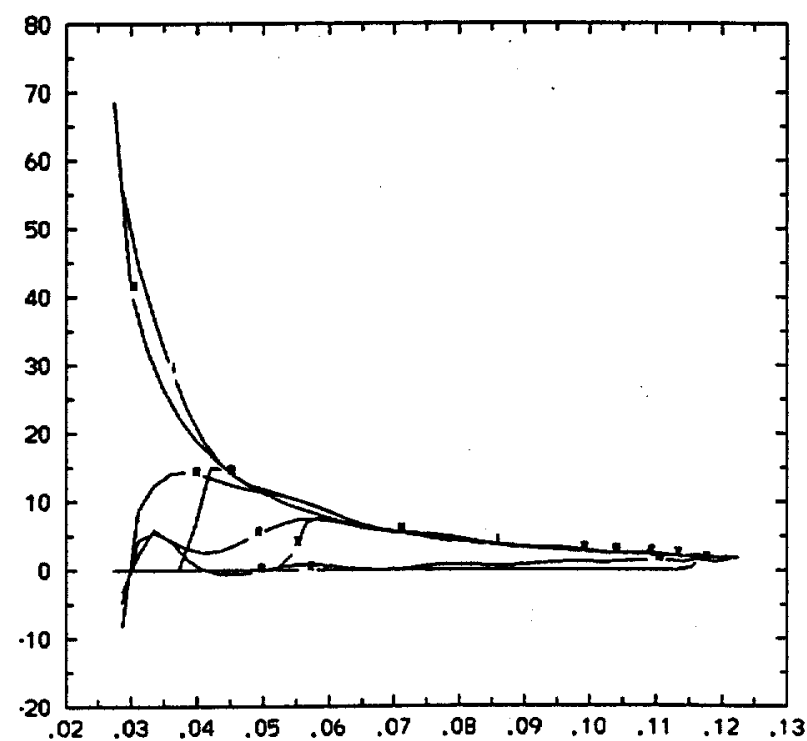

Figure 5. Spherical Streaming problem, radiation energy density vs. radius; analytic and $\mathbf{P n}$ computational solutions at steady-state and various times after source turns off.

The first set of plots shows the analytic solution for radiation energy density as a function of distance.The following set of plots show the radiation energy densities as a function of distance, computed with the
Pn code. The computed solution is rather far from the analytic solution, especially near the inner sphere.

The discontinuous finite element and Sn methods get reasonable results for this problem. The following plots show that they both compute the steady-state energy density solution very accurately, and smear out the transient solution. However, the finite element method is noticeably more accurate near the sharp transitions where the source is seen to be turning off.

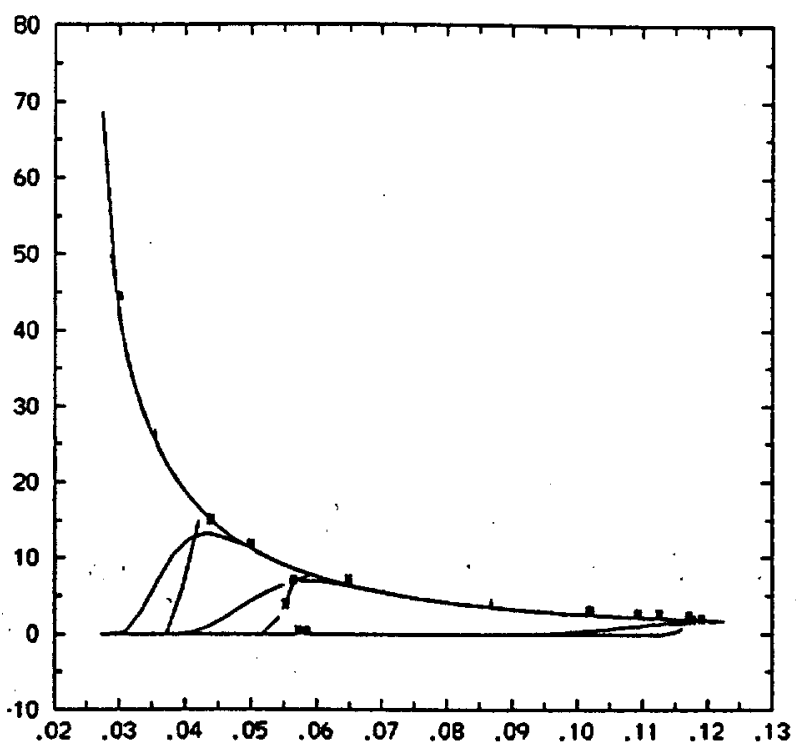

Figure 6. Spherical Streaming problem, radiation energy density vs. radius; analytic and finite element computational solutions at steady-state and various times after source turns off.

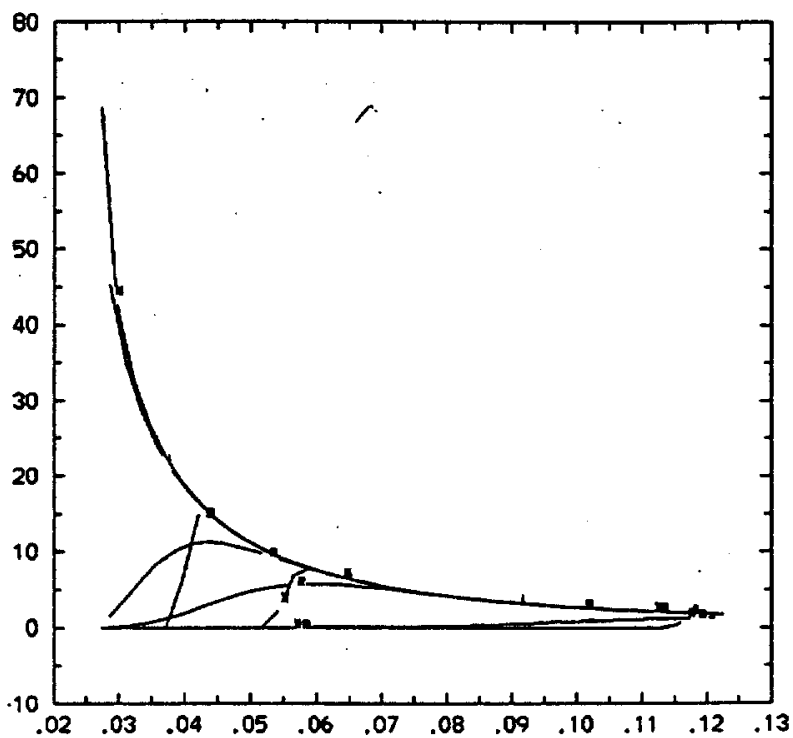

Figure 7. Spherical Streaming problem, radiation energy density vs. radius; analytic and Sn computational solutions at steady-state and various times after source turns off.

The story is different when we look at directional dependence. The next plots show intensity as a function of angle, in one zone. The steady-state solution is a step function, as was discussed above. The discontinuous finite element solution captures the step in three zones, with a slight Gibbs oscillation. The Sn solution smears 
it out enough to obscure the qualitative character of the solution.
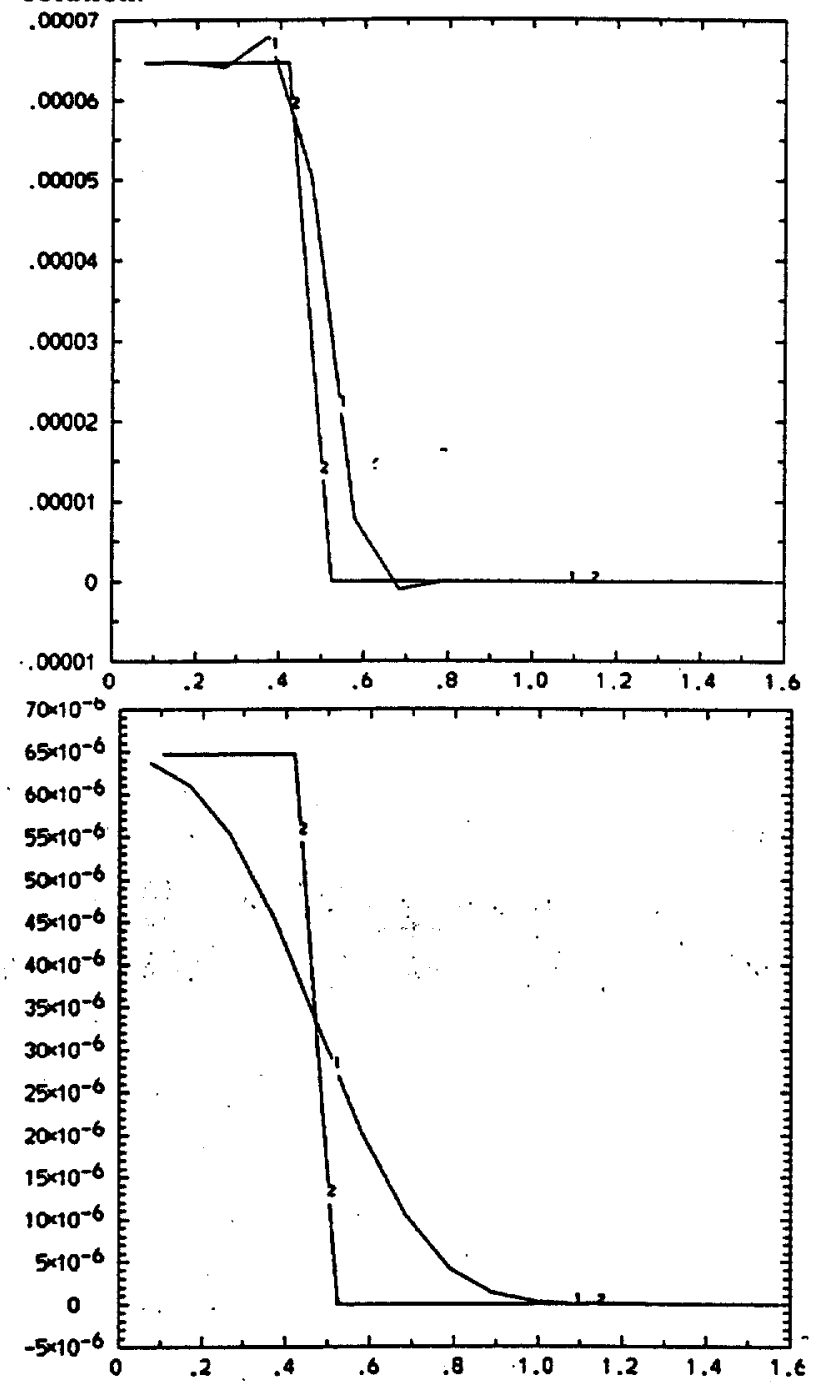

Figure 8. Zonal radiation intensity as a function of angle, at one zone, with source on. Upper plots: analytic and finite element computational solutions. Lower plots: analytic and Sn computational solutions.

Shortly after the source is turned off, it looks like an annulus from a fixed zone: the central part of the sphere looks dark because it is close enough so that the last radiation it emitted has already passed by. The following plots show the solutions at that time. The analytic solution the in the plots is that for the center of the zone; the position of the inner (left) jump varies widely through the zone. The Sn solution entirely misses the dark center. The finite element solution picks it up, although it is smeared out.

The three methods compare in computer time per cycle roughly as in the thermal wave problem.
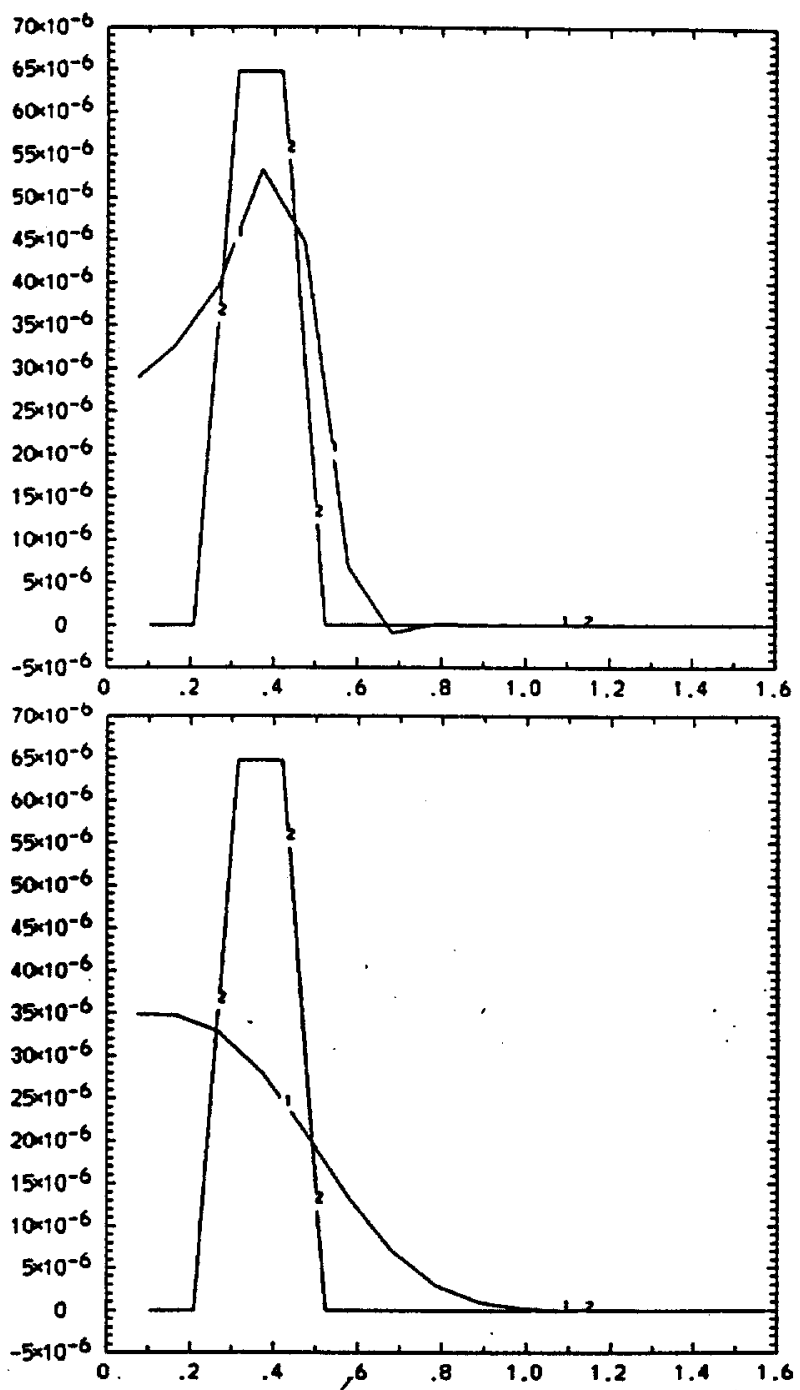

Figure 9. Zonal radiation intensity as a function of angle, at one zone, shortly after source turns off. Upper plots: analytic and finite element computational solutions. Lower plots: analytic and Sn computational solutions.

\section{References}

Kershaw, David, and Harte, Judith, $2 D$

Deterministic Radiation Transport with the

Discontinuous Finite Element Method, Lawrence Livermore National Laboratory, Livermore, CA, UCRL-ID-115525 (1993).

Lund, C. M. and Wilson, J. R., Some Numerical Methods for Time-Dependent Multifrequency Radiation Transport Calculations in One Dimension, Lawrence Livermore National Laboratory, Livermore, CA, UCRL-84678 (1980). 


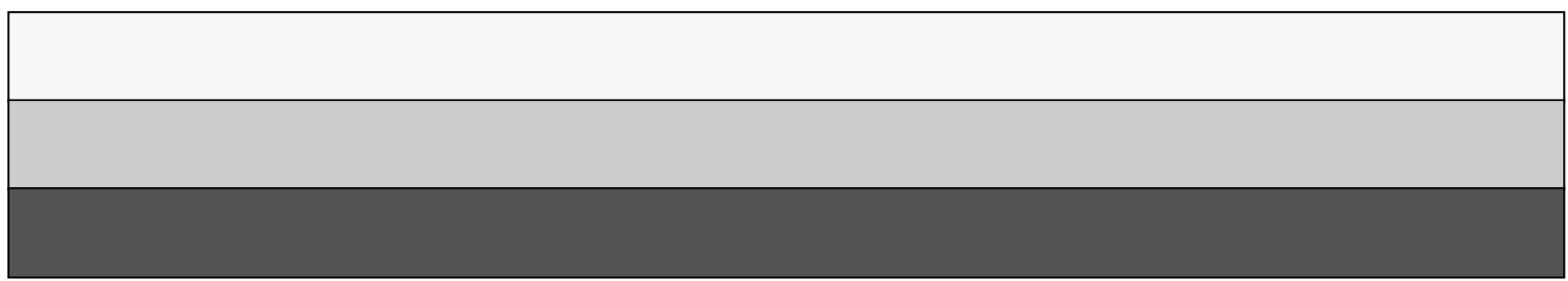

